\title{
Adult presentation of congenital cystic adenomatoid malformation: Successful surgical management
}

\author{
Andrew R. Davies, MD, MRCS, Vinayak Bapat, MD, FRCS, and
}

Tom Treasure, MD, FRCS, London, United Kingdom

Supplemental material is available online.

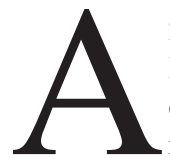

24-year-old Nigerian woman, resident in the United Kingdom, collapsed on a plane but quickly recovered with basic resuscitation and oxygen. She arrived safely in Nigeria, did not seek medical advice, and enjoyed her holiday. On returning to the United Kingdom, she had 2 admissions to the Accident \& Emergency division of Guy's Hospital 6 weeks apart.

On initial presentation with dyspnea and pleuritic chest pain, she was found to have reduced air entry on the left and, after a chest radiograph, was treated for primary spontaneous pneumothorax (needle thoracocentesis) before discharge. On representation and further radiography, she underwent formal chest drainage, which was stopped 5 days later because of apparent improvement. No radiograph was obtained after drain removal.

At this point, she was referred to our unit with a diagnosis of recurrent spontaneous pneumothorax for consideration of videoassisted thoracoscopy.

In the surgical clinic she told us that she had been given a diagnosis of asthma, but what she described was exertional dyspnea. She was a nonsmoker.

On review of her radiographs (Figure 1), we were suspicious that they were more consistent with giant bullous disease than primary spontaneous pneumothorax. Computed tomographic scanning (Figures 2, E1, and E2) revealed an unexpected and extremely rare cause for her presentation. A senior radiologist reported multiple cystic lesions (consistent with congenital cystic adenomatoid malformation [CCAM]) and interpreted that they were largely replacing the lower lobe. The remaining lung was significantly compressed. After discussion, we planned for left lower lobectomy and possible upper lobe resection, depending on intraoperative findings.

From the Department of Thoracic Surgery, Guy's Hospital, London, United Kingdom.

Presented at the Royal Society of Medicine, Cardiothoracic Section, Nov 2004.

Received for publication April 27, 2006; revisions received May 21, 2006; accepted for publication June 7, 2006.

Address for reprints: Tom Treasure, MD, Department of Thoracic Surgery, Guy's Hospital, London SE1 9RT, United Kingdom (E-mail: tom.treasure@ ukgateway.net)

J Thorac Cardiovasc Surg 2006;132:1493-4

$0022-5223 / \$ 32.00$

Copyright () 2006 by The American Association for Thoracic Surgery

doi:10.1016/j.jtcvs.2006.06.041
At thoracotomy, we found multiple, thick-walled, gelatinous cystic lesions occupying the left hemithorax. Surprisingly, these appeared to be originating within the fissure and had not replaced the lobe itself. Fortunately, this was

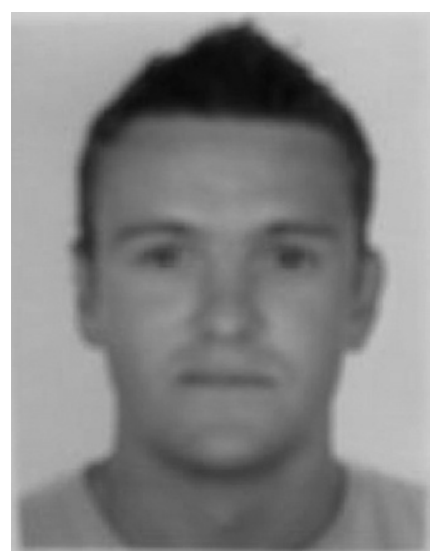

Dr Davies appreciated before any vessels were divided. At the conclusion of dissection, both lobes reinflated and appeared macroscopically normal. Despite a small air leak, she had a good recovery. During an outpatient visit 3 weeks later, she reported a considerable improvement in her symptoms. Histopathology confirmed type II CCAM.

CCAM was first described in 1949. It is a developmental hamartomatous abnormality of the lung with adenomatoid cyst proliferation. ${ }^{1}$

It is believed to result from aberrant embryogenesis at or before the seventh gestational week, producing adenomatous proliferation of the terminal bronchioles and cystic distortion of the lung architecture. Blood supply is through the pulmonary circulation.

The cause of CCAM is unknown, but the condition nearly always presents prenatally (incidentally picked up on prenatal ultrasonography) or within the first year of life, manifesting as respiratory distress in neonates or recurrent infections in older children. $^{2}$

Adult presentation of CCAM is very rare, with few cases documented in the literature. ${ }^{3-5}$ Mildly affected individuals can remain asymptomatic, with the disease even being diagnosed postmortem in elderly patients who have died from an unrelated illness.

The Stocker classification subdivides CCAM according to the size of the cysts and other histologic criteria:

- Type 1: one or more cysts (2-10 cm diameter) with mucinogenic differentiation. This is the most common form.

- Type 2: Smaller cysts (classically $0.5-2 \mathrm{~cm}$ ) lined by cuboid or columnar epithelium.

- Type 3: Microscopic adenomatoid cysts. These macroscopically might appear to be a solid mass.

The prognosis of CCAM is very variable. Overall mortality is quoted at $25 \%$ to $30 \%$ but might approach $100 \%$ in severely affected individuals without treatment. There is a spectrum of severity through to the asymptomatic adult. Type 3 CCAM carries the worst prognosis but fortunately is the least common. ${ }^{1}$

Treatment is primarily surgical, and although some specialist centers are now undertaking fetal surgery (predominantly indicated for those with hydrops fetalis), most documented postnatal intervention has centered around lung resection (in the form of lobectomy/pneumonectomy) to remove the lesion and relieve com- 


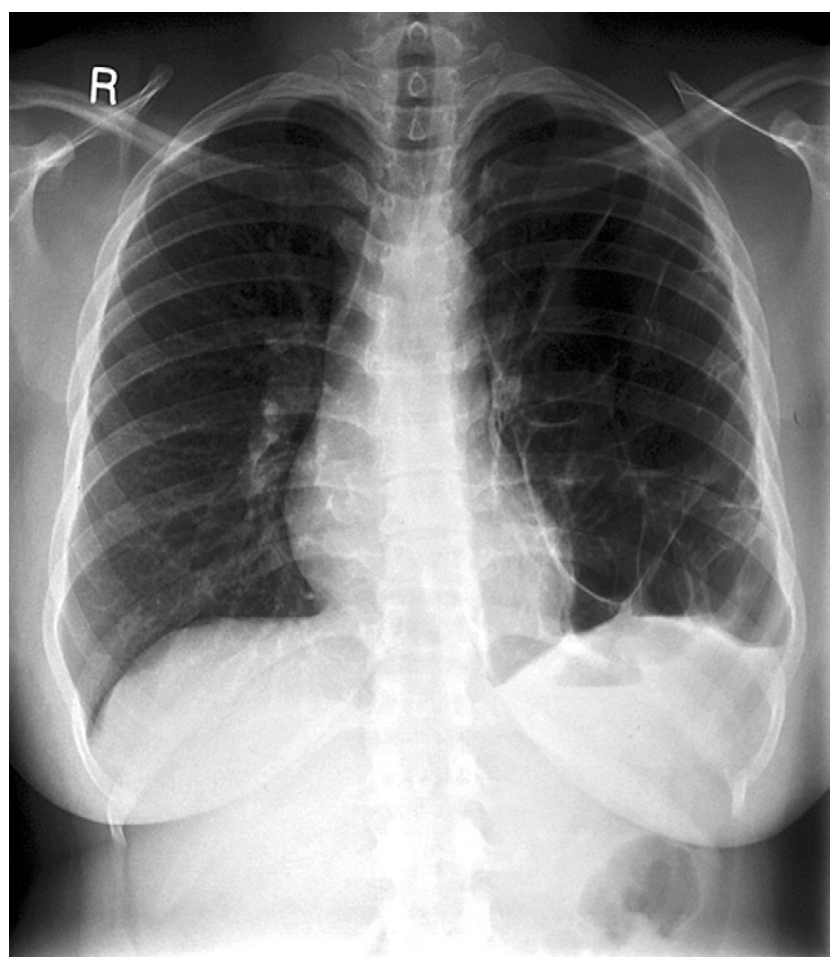

Figure 1. Chest $x$-ray demonstrating multiple cystic lesions consistent with CCAM. CCAM, congenital cystic adenomatoid malformation.

pression of the residual lung. This initially appeared to be merited on the basis of radiologic imaging.

The advantage of surgical intervention is that it also prevents the development of secondary complications of CCAM, namely hemorrhage and recurrent infection, and the development of malignancy. ${ }^{2}$ Rhabdomyosarcomas, pulmonary blastomas, bronchioloalveolar carcinomas, and squamous cell carcinomas have all been described.

CCAM (especially type 2 CCAM, as in this case) is associated with other congenital anomalies predominantly affecting the renal, gastrointestinal, and cardiac systems. It is important that such patients be routinely screened to anticipate any further problems before they manifest clinically.

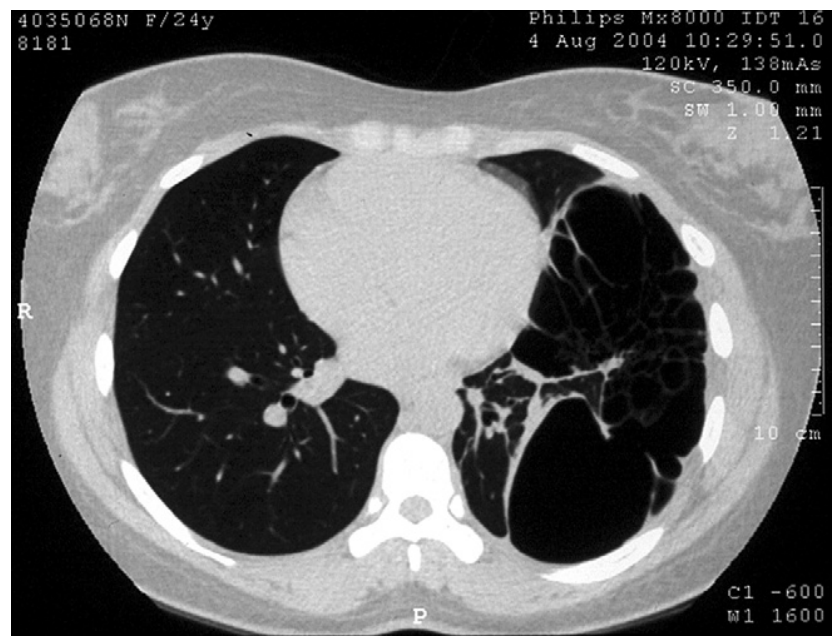

Figure 2. Chest computed tomogram demonstrating multiple cystic lesions consistent with CCAM. CCAM, congenital cystic adenomatoid malformation.

This case highlights a rare presentation of CCAM in an adult. It is also testament to the fact that unusual conditions should be managed on an individual basis because premeditated lobectomy (the treatment of choice for previously documented cases of CCAM and merited on imaging) would have resulted in an unnecessary lung resection in this patient.

\section{References}

1. Mandell G. Congenital cystic adenomatoid malformation. eMedicine review, Jan 10, 2003. Available at: www.emedicine.com/radio/topic186. htm. Accessed 10 May 2006

2. Vaughan DJ. Cystic adenomatoid malformation. eMedicine review, Nov 15, 2002. Available at: www.emedicine.com/ped/topic534.htm. Accessed 10 May 2006.

3. Plit ML. Clinical, radiographic and lung function features of diffuse congenital cystic adenomatoid malformation of the lung in an adult. Eur Respir J. 1997;10:1680-2.

4. Han YM, Lee DK, Lee SY, Chung KH, Kim MH, Sohn MH, et al. Adult presentation of congenital cystic adenomatoid malformation of the lung: a case report. J Korean Med Sci. 1994;9:86-91.

5. Avitabile AM. Congenital cystic adenomatoid malformation of the lung in adults. Am J Surg Pathol. 1984;8:193-202. 


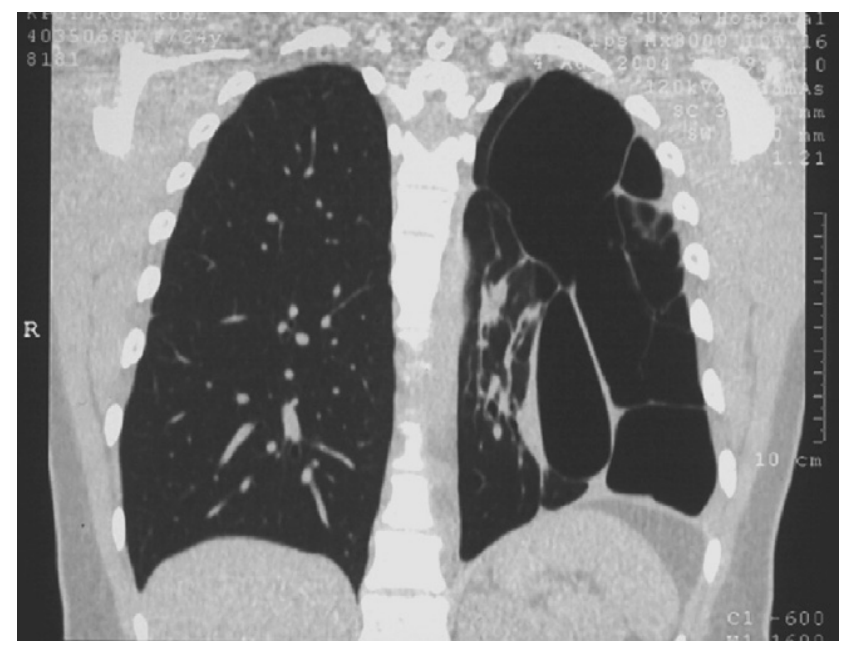

Figure E1. Coronal computed to mogram showing extensive replacement of left lung with cystic lesions.

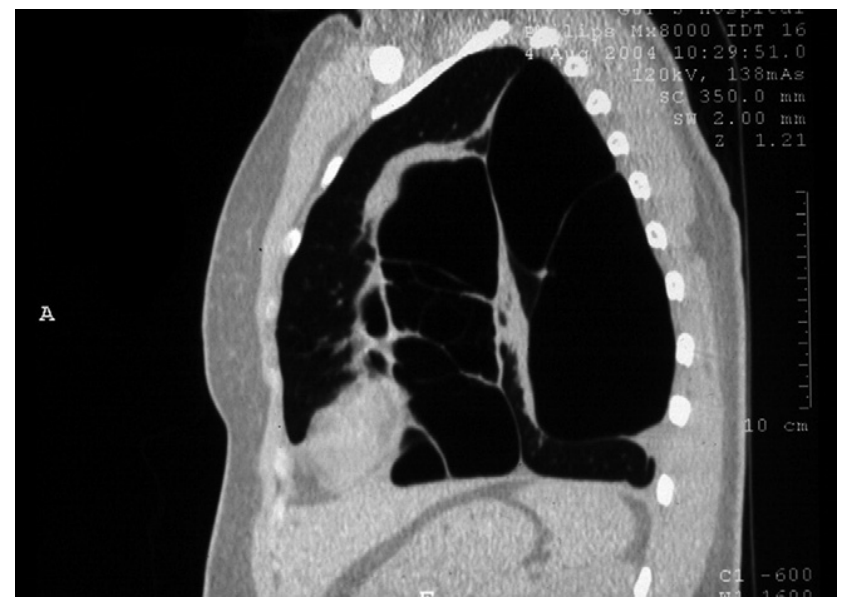

Figure E2. Sagittal chest computed tomogram demonstrating some residual upper and lower lobe lung parenchyma. 